\title{
Interactions between Tandem Cylinders in an Open Channel: Impact on Mean and Turbulent Flow Characteristics
}

\author{
Hasan Zobeyer ${ }^{1}{ }^{*}$, Abul B. M. Baki ${ }^{2}$ and Saika Nowshin Nowrin ${ }^{1}$ \\ 1 Department of Water Resources Engineering, Bangladesh University of Engineering and Technology, \\ Dhaka 1000, Bangladesh; nowshin.saika@gmail.com \\ 2 Department of Civil and Environmental Engineering, Clarkson University, Potsdam, NY 13699, USA; \\ abaki@clarkson.edu \\ * Correspondence: zobeyer@wre.buet.ac.bd
}

Citation: Zobeyer, H.; Baki, A.B.M.; Nowrin, S.N. Interactions between Tandem Cylinders in an Open Channel: Impact on Mean and Turbulent Flow Characteristics. Water 2021, 13, 1718. https://doi.org/ 10.3390/w13131718

Academic Editor:

Bommanna Krishnappan

Received: 5 May 2021

Accepted: 18 June 2021

Published: 22 June 2021

Publisher's Note: MDPI stays neutral with regard to jurisdictional claims in published maps and institutional affiliations.

Copyright: (c) 2021 by the authors. Licensee MDPI, Basel, Switzerland. This article is an open access article distributed under the terms and conditions of the Creative Commons Attribution (CC BY) license (https:/ / creativecommons.org/licenses/by/ $4.0 /)$.

\begin{abstract}
The flow hydrodynamics around a single cylinder differ significantly from the flow fields around two cylinders in a tandem or side-by-side arrangement. In this study, the experimental results on the mean and turbulence characteristics of flow generated by a pair of cylinders placed in tandem in an open-channel flume are presented. An acoustic Doppler velocimeter (ADV) was used to measure the instantaneous three-dimensional velocity components. This study investigated the effect of cylinder spacing at 3D, 6D, and 9D (center to center) distances on the mean and turbulent flow profiles and the distribution of near-bed shear stress behind the tandem cylinders in the plane of symmetry, where $\mathrm{D}$ is the cylinder diameter. The results revealed that the downstream cylinder influenced the flow development between cylinders (i.e., midstream) with 3D, 6D, and 9D spacing. However, the downstream cylinder controlled the flow recirculation length midstream for the 3D distance and showed zero interruption in the 6D and 9D distances. The peak of the turbulent metrics generally occurred near the end of the recirculation zone in all scenarios.
\end{abstract}

Keywords: mean and turbulent flow characteristics; near-bed shear stress; open channel flow; tandem cylinders

\section{Introduction}

Cylinders in tandem or side-by-side arrangements provide the simplest configurations of multiple slender structures in engineering projects (e.g., bridge piers, offshore and onshore structures, oil and gas pipelines, power lines, high-rise buildings, fish habitat structures, and flow past vegetation) [1,2]. Therefore, the hydrodynamic interference between two cylinders provides a good model for gaining insight into the complex flow characters around more cylinders, and has drawn considerable interest from researchers [3,4].

The significance of fluid flow around a single infinite/finite cylinder has been investigated for decades and has been widely studied in the literature [3]. Understanding the flow field around a single cylinder is not sufficient for understanding the interactions between cylinders in various configurations [2] that are encountered in practice. The flow characteristics over a single cylinder differ significantly from the flow field downstream of two cylinders due to the interaction between the individual cylinders [4]. Nosier et al. [3] highlighted that the interaction between cylinders affects the flow behavior and vortex shedding and, consequently, the forces acting upon the cylinders. A distinct flow pattern was observed when changing the finite cylinder's height, differing from the infinite cylinder case [5-7]. The generated tip vortex structures near the free end of the submerged cylinder influence the near/far wake flow fields [3].

Since the earliest study by Zdravkovich [8], several numerical $([4,9,10]$, among others) and experimental studies $[1,3,11,12]$ have investigated the flow passing through the infinite cylinders in a tandem arrangement with low to high Reynolds numbers (an in-depth review is provided by Heidari [2]). They found that the flow field in tandem cylinders 
is significantly influenced by the Reynolds number and the gap ratio $(L / D)$, where $L$ is the distance between two cylinders (center to center, $\mathrm{c} / \mathrm{c}$ ) and $D$ is the diameter of the cylinder. Several studies (e.g., $[1,12]$ for $1.5<L / D>6$ at $\left.R e_{\mathrm{D}}=800-42,000\right)$ reported three flow regimes: the extended-body regime $(L / D<2$, vortex street far behind the downstream cylinder), the reattachment regime $(L / D=2-5$, shed vortices from upstream cylinder formed just behind the downstream cylinder), and the co-shedding regime $(L / D>5$, where each cylinder sheds its own von Kármán vortices individually). The studies mentioned so far focused on the effects of cylinder spacing and Reynolds number on vortex development and movement in the wake.

Previous investigations have improved our understanding of the flow field around wall-mounted cylinders in a tandem arrangement. However, many issues remain unresolved. For example: How do the detailed mean and turbulent flow profiles and their development behind the tandem cylinders depend on $L / D$ ? Are the flow fields behind the cylinders at $L / D=3$ and 6 different from that at $L / D=9$ ? What is the distribution of near-bed shear stress behind the cylinders at different values of $L / D$ ? Therefore, to provide a better physical understanding, the key goal of this study was to experimentally investigate the flow characteristics around emergent cylinders in a tandem arrangement. In meeting this study's goal, one objective was to study the effect of $L / D$ on the mean and turbulent flow profiles behind the tandem cylinders in the plane of symmetry. There is a lack of information on the mean and turbulent flow profiles in the literature. The second objective was to investigate how the presence of a downstream cylinder affects the flow fields in-between cylinders. The third objective was to examine the effect of $L / D$ on the distribution of near-bed shear stress behind the cylinders.

The findings of this study could be used to improve knowledge on flow profiles and near-bed shear stress in the aspect of local sediment transport behind cylinders in a tandem arrangement and may help to improve the functionality of the cylinder structures in water resource engineering applications (e.g., bridge piers, offshore and onshore structures, fish habitat structures).

\section{Materials and Methods}

\subsection{Experimental Setup}

Experiments were performed in a $21.3 \mathrm{~m}$ long, $0.76 \mathrm{~m}$ wide, and $0.74 \mathrm{~m}$ deep recirculating rectangular flume with an adjustable slope. For the narrow open channel, the side wall effects were assumed to be negligible based on some preliminary analyses on velocity distribution throughout the width of the flume. The flume sidewalls were transparent for visual access to the flow, and the longitudinal bed slope was set to $1.87 \times 10^{-3}$. Two solid concrete circular cylinders of equal height $\left(H_{\mathrm{d}}=0.30 \mathrm{~m}\right)$ and diameter $(D=0.15 \mathrm{~m})$ were mounted vertically along the channel centerline of a smooth flume bed at a distance of $11.0 \mathrm{~m}$ from the inlet to ensure that the turbulent flow was fully developed in the test section (the approach flow development is discussed in Section 3.1.). Then, 3 experimental scenarios (hereafter called S1, S2, and S3) were performed, where the cylinders were aligned in a tandem arrangement (Figure 1) with 3 different gap ratios of $L / D=3 \mathrm{D}$ in $\mathrm{S} 1$, $L / D=6 \mathrm{D}$ in $\mathrm{S} 2$, and $L / D=9 \mathrm{D}$ in S3 center to center, with a flow blockage ratio of $20 \%$. We acknowledge that there may have been some effects of cylinder blockage on the flow characteristics in a narrow channel. Nevertheless, because the main objective in this study was related to flow characteristics along the centerline (behind the tandem cylinders in the plane of symmetry), this blockage ratio was not expected to affect the key findings of this study significantly. Further studies are recommended to examine the effect of blockage ratio on flow characteristics for tandem cylinders.

In the 3 scenarios, the same approach uniform flow condition was maintained, where the approach flow depth was $H=26.5 \mathrm{~cm}$, and the depth-averaged flow velocity was $\bar{U}_{0}=35.3 \mathrm{~cm} / \mathrm{s}$ (Table 1$)$. The cylinder's aspect ratio was $H / D=1.80$. Some shallow flow studies were conducted using similar $H / D$ values (e.g., $H / D=0.5,1.0,2.0$ by Akilli and Rockwell [13], $H / D=1.9$ by Sadeque et al. [6], $H / D=1.25$ by Gao et al. [14], and $H / D=1.12$ 
by Kirkil and Constantinescu [15,16]). The approach shear velocity was $u *=1.40 \mathrm{~cm} / \mathrm{s}$, estimated by the Reynolds shear stress method $\left(\tau=\rho u_{*}^{2}(1-z / \sigma) \cong-\rho \overline{u^{\prime} w^{\prime}}\right)$ by Nezu and Nakagawa [17], using the measured Reynolds shear stress very close to the bed (10\% depth from the bed), where $\tau$ is the total shear stress, $\sigma$ is the distance from the bottom to the point of the maximum velocity, $\rho$ is the water density, and $u^{\prime}$ and $w^{\prime}$ are the velocity fluctuations in the streamwise and vertical directions, respectively. All experiments were conducted at higher Reynolds numbers based on the diameter of the cylinder, $R e_{D}=\frac{\bar{U}_{0} D}{\vartheta}=52,900$, where $\vartheta$ is the coefficient of kinematic viscosity of water.
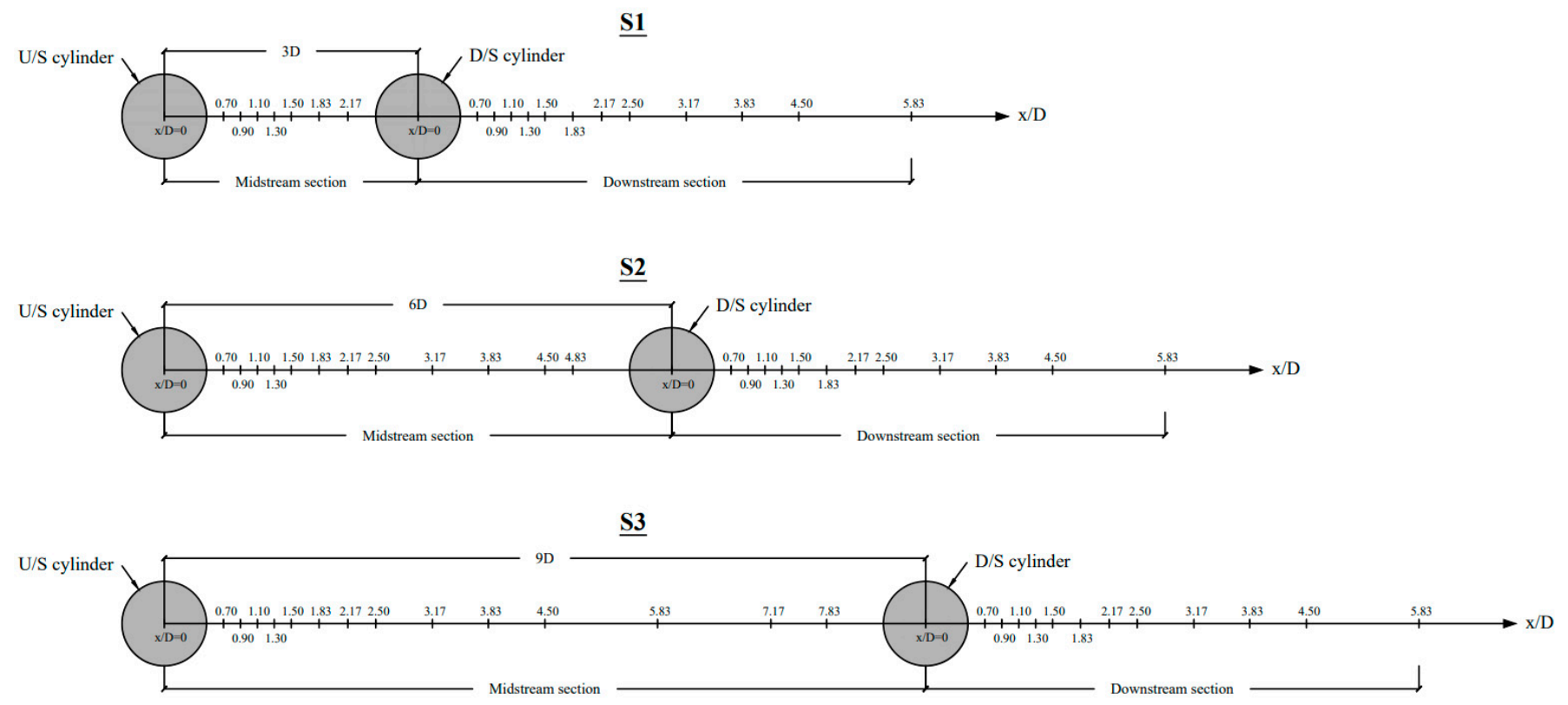

Figure 1. Plan view of the position of the measurement stations in the symmetry plane midstream and downstream for 3 scenarios: S1, S2, and S3. The + symbol shows the position of the measurement stations. U/S and D/S indicate upstream and downstream, respectively.

Table 1. Details regarding the experimental scenarios.

\begin{tabular}{|c|c|c|c|c|c|c|c|c|c|c|}
\hline $\begin{array}{l}\text { Experimental } \\
\text { Scenarios }\end{array}$ & $\begin{array}{c}\text { Cylinder } \\
\text { Diameter }(D)\end{array}$ & $\begin{array}{l}\text { Cylinder } \\
\text { Spacing * }\end{array}$ & $\begin{array}{l}\text { Flow } \\
\text { Rate }\end{array}$ & $\begin{array}{c}\text { Approach } \\
\text { Water Depth } \\
(H)\end{array}$ & $\begin{array}{l}\text { Approach Flow } \\
\text { Velocity }^{\ominus}\end{array}$ & $\begin{array}{c}\text { Reynolds } \\
\text { Number } \mp \\
\left(R_{d}\right)\end{array}$ & $\begin{array}{c}\text { Shear } \\
\text { Velocity } \pm \\
\left(u^{*}\right)\end{array}$ & $\begin{array}{c}\text { Average } \\
\text { COR }\end{array}$ & $\begin{array}{c}\text { Average } \\
\text { SNR }\end{array}$ & $\begin{array}{c}\text { Data } \\
\text { Retained }\end{array}$ \\
\hline- & $(\mathrm{cm})$ & (times) & $\left(\mathrm{m}^{3} / \mathrm{s}\right)$ & $(\mathrm{cm})$ & $(\mathrm{cm} / \mathrm{s})$ & - & $(\mathrm{cm} / \mathrm{s})$ & $(\%)$ & $(\mathrm{dB})$ & $(\%)$ \\
\hline S1 & 15 & $3 \mathrm{D}$ & 0.069 & 26.5 & 35.3 & 52,900 & 1.4 & 80.6 & 16.7 & 90.1 \\
\hline S2 & 15 & $6 \mathrm{D}$ & 0.069 & 26.5 & 35.3 & 52,900 & 1.4 & 80.6 & 16.9 & 90.4 \\
\hline S3 & 15 & 9D & 0.069 & 26.5 & 35.3 & 52,900 & 1.4 & 78.3 & 15.6 & 91.2 \\
\hline
\end{tabular}

\pm The Reynolds shear stress method was applied to estimate $\mathrm{u} *$ using measured Reynolds shear stress very close to the bed (lower $10 \%$ depth). + Reynolds number is based on the diameter of the cylinder. ${ }^{*}$ Spacing is in center to center. ${ }^{\theta}$ Depth-averaged approach flow velocity magnitude.

Figure 1 illustrates the position of the measurement stations in the symmetry plane between cylinders (hereafter referred to as "midstream") and downstream of the second cylinder (referred to as "downstream"). Midstream, the measurement stations at various relative streamwise distances varied from $x / D=0.70$ to 2.17 in $S 1, x / D=0.70$ to 4.83 in $\mathrm{S} 2$, and $x / D=0.70$ to 7.83 in $S 3$; and downstream the stations were at $x / D=0.70,0.90,1.10,1.30$, $1.50,1.83,2.17,2.50,3.17,3.83,4.50$, and 5.83, and were the same in all scenarios (Figure 1). At each station both midstream and downstream, the vertical distance $(z)$ between 2 points varied from 0.5 to $5 \mathrm{~cm}$, and the relative depth $(z / H)$ of the measured points varied from 0.02 to 0.75 , covering both the inner layer ( $20 \%$ of flow depth) and outer layer (remaining $80 \%$ of flow depth) $[17,18]$. The near-bed measurement points were $0.5 \mathrm{~cm}(z / H=0.02)$ 
away from the bed. Some other studies also took near-bed measurements at distances similar to those of this study $[19,20]$.

\subsection{ADV Measurements and Analysis}

A downward-facing acoustic Doppler velocimeter (ADV) (SonTek, 16-Hz MicroADV) was used to measure the 3D instantaneous velocity time-series data at each point. Measurements were recorded at a rate of $30 \mathrm{~Hz}$ for a period of $120 \mathrm{~s}$ at each point, with a sampling volume of $0.05 \mathrm{~cm}^{3}$ and assuming implicitly that such a duration was long enough to describe the turbulence. Some studies used relatively small number of samples for turbulence measurements, using ADVs as low as 3000 samples [20] and 1500 samples [19]. The raw data were first post-processed using WinADV [21] to remove spikes using the phase space threshold method of Goring and Nikora [22]. Velocity signal correlations (COR) and the signal-to-noise ratio (SNR) are commonly used to eliminate poor-quality data from measured time series. A filtering scheme with an average COR $\leq 70 \%$ and average SNR $\leq 15 \mathrm{~dB}$ was used to eliminate bad data from the velocity time series to yield highly reliable data [20]. Such criteria led to more than $90 \%$ of the data being retained in each experimental scenario (Table 1). Afterward, the velocity time series in 3 directions that had noisy signals (velocity spectra that generally displayed a flat slope instead of the Kolmogorov $-5 / 3$ slope in the inertial subrange) were removed by visual inspection, thus rejecting about $10 \%$ of data points. It should be noted that due to the limitations of the downward-facing ADV, flow information for the top $6.5 \mathrm{~cm}$ of the flow depth is not available.

The time-averaged mean and turbulence metrics were estimated at each measurement point/location. The vertical profile of time-averaged velocity in the streamwise direction $(u)$ was compared with the classical logarithmic law for the hydraulically rough flow regime:

$$
\frac{u}{u_{*}}=\frac{1}{\kappa} \operatorname{In}\left(\frac{z}{k}\right)+8.5
$$

where $\kappa$ is the von Karman constant $(=0.41)$ and $k$ is the medium bed roughness height ( $k=0.0105 \mathrm{~m}$, equivalent to Manning's $n=0.015$ for smooth cast iron bottom). We assumed that the logarithmic law described the velocity distribution over the entire depth of uniform and steady open-channel flows in practical applications.

The turbulence metrics included turbulent kinetic energy $\left(k_{e}=0.5\left(\overline{u^{\prime 2}}+\overline{v^{\prime 2}}+\overline{w^{\prime 2}}\right)\right.$ ) and the resultant Reynolds shear stress $\left(\tau_{p}\right)$ per unit mass density of fluid on a surface parallel to the bed:

$$
\tau_{p}=\left[\left(-\overline{u^{\prime} w^{\prime}}\right)^{2}+\left(\overline{-v^{\prime} w^{\prime}}\right)^{2}\right]^{1 / 2}
$$

where $v^{\prime}$ is the velocity fluctuations in the transverse direction, $-\left(\overline{u^{\prime} w^{\prime}}\right)$ is the primary Reynolds shear stress (per unit mass density of fluid) on the vertical plane $(x-z)$, and $-\left(\overline{v^{\prime} w^{\prime}}\right)$ is the Reynolds shear stress (per unit mass density of fluid) on the transverse plane $(y-z)$; the bar sign over $u \prime w \prime$ and $v^{\prime} w^{\prime}$ indicates a time-averaged value. Herein, $\tau_{p}$ was estimated leveraging the Reynolds shear stress in both vertical and transverse planes instead of using the measured Reynolds shear stress on the horizontal plane.

The dimensionless near bed shear stress $\left(\tau_{b}^{*}\right)$ was estimated from 3 different Reynolds, TKE, and modified TKE methods. In Reynolds method, the dimensionless bed shear stress was estimated from the resultant Reynolds shear stress:

$$
\tau_{b}^{*}=\frac{\tau_{p}}{u_{*}^{2}}
$$


Using the turbulent kinetic energy (TKE) method, the dimensionless bed shear stress was estimated as below [23]:

$$
\tau_{b}^{*}=\frac{C_{1} k_{e}}{u_{*}^{2}}
$$

where $C_{1}$ is a constant equal to 0.19 [24]. The modified TKE method only uses the vertical component of the velocity fluctuations due to the smaller noise of the measurement instrument in this direction [24]. Therefore, the dimensionless bed shear stress (per unit mass density of fluid) was estimated as below:

$$
\tau_{b}^{*}=\frac{C_{2} w_{r m s}^{\prime}}{u_{*}^{2}}=C_{2} \overline{w^{\prime 2}} / u_{*}^{2}
$$

where $C_{2}$ is a constant equal to 0.9 [24]. The near-bed shear stress at each station was estimated using Equations (3)-(5) for all points within the inner layer $(20 \%$ of the flow depth from the bed) in all scenarios. However, the maximum near-bed shear stress values out of several points within the inner layer are presented.

\section{Results and Discussion}

\subsection{Mean Flow Characteristics}

The mean flow characteristics were analyzed to better understand the flow fields in both midstream and downstream in terms of variation of velocity profiles, size of recirculation zone, and the velocity development process. Figure 2 shows the vertical profiles of dimensionless streamwise velocity $\left(u / u^{*}\right)$ in the plane of symmetry for all three scenarios both midstream and downstream. The Figure confirms that the vertical profiles of the streamwise approach velocity satisfy the logarithmic law (Equation (1)), assuring a fully developed approach flow for a hydraulically rough flow regime.

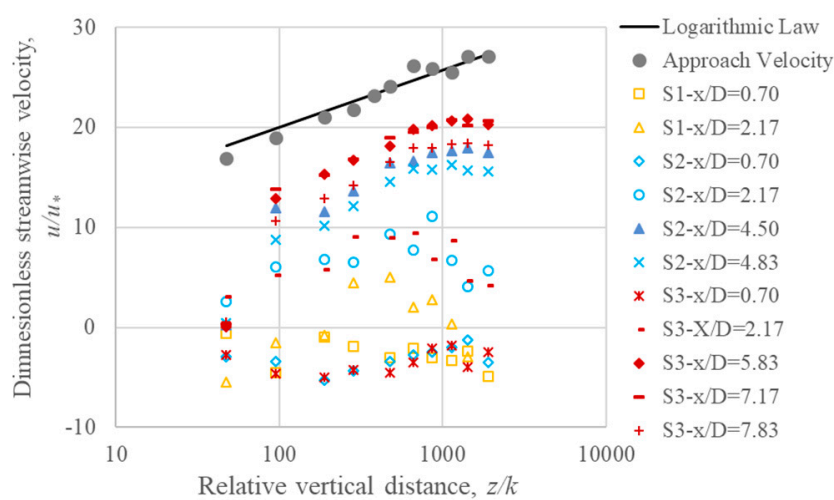

(a)

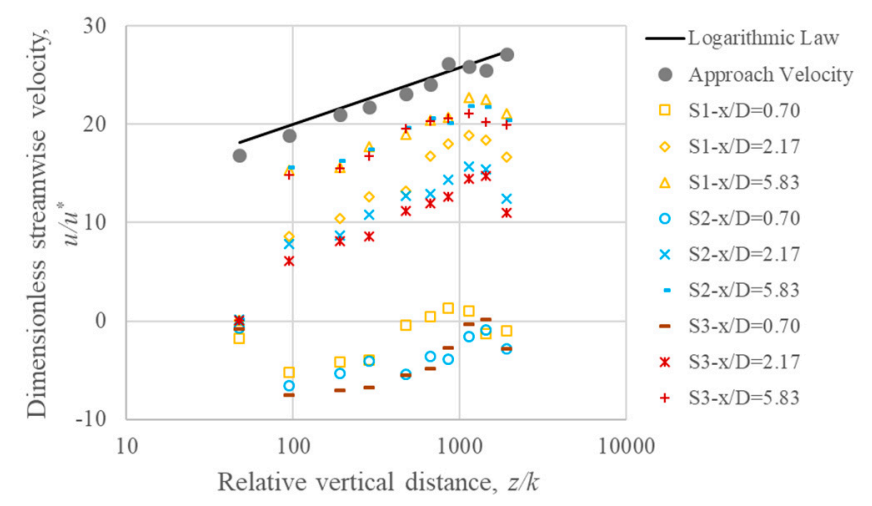

(b)

Figure 2. Vertical profiles of dimensionless streamwise velocity $\left(u / u^{*}\right)$ in the symmetry plane: (a) midstream and (b) downstream. A solid black line shows the classical logarithmic law.

Midstream, all the velocity profiles for all scenarios deviated from the logarithmic law (Figure 2a), as expected because of flow disturbances caused by the cylinders. The profiles at $x / D=0.70$ showed significant and maximum deviations from the logarithmic profile in all scenarios. The deviations became smaller with increasing downstream distance from the cylinder; the smallest average deviations were of about $95 \%$ in $\mathrm{S} 1$ (at $x / D=2.17$ ), 36\% in S2 (at $x / D=4.50)$, and $24 \%($ at $x / D=7.17)$ in S3. In S3, the profile at $x / D=7.17$ was the closest to the logarithmic profile, as compared to that at $x / D=7.83$ (34\% deviation), where the profiles were influenced by the downstream cylinder.

Similarly, downstream in all scenarios the profiles at $x / D=0.70$ showed maximum deviations from the logarithmic profile, and the deviations became smaller with the downstream distance from the cylinder (Figure $2 \mathrm{~b}$ ). The profiles at $x / D=5.83$ were the closest to 
the logarithmic profile $(20 \%, 21 \%$, and $22 \%$ deviations in S1, S2, and S3, respectively), approaching a fully developed undisturbed flow. Downstream in S3, the profiles at $x / D=5.83$ were closer (about $6 \%$ ) to the logarithmic profile as compared to the midstream profiles between $x / D=5.83$ and 7.83 . Therefore, it was revealed that the rate of flow development in downstream was faster than that midstream, providing further evidence that the downstream cylinder influenced the midstream profiles in all scenarios.

Figure 3 shows the velocity vector and isocontours of dimensionless streamwise velocity $\left(u / u_{*}\right)$ in the symmetry plane both midstream and downstream for all three scenarios. Midstream, based on velocity vector and $u / u_{*} \leq 0$, the flow recirculation length on the symmetry plane was $x / D \sim 1.80$ in S1, and $\sim 1.50$ in S2 and S3 almost over the entire depth, and was stronger near the free surface than the bed level. On the other hand, downstream, flow recirculation was observed immediately behind the cylinder and was strong near the bed level. The size of the recirculation was $x / D \sim 0.90$ in S1, 1.10 in S2, and 1.30 in S3, with values much smaller and weaker than that midstream. For an emergent cylinder and high Reynolds number, Sadeque et al. [6] determined $x / D \sim 1.50$ for the entire water depth and for the rough bed; Kirkil and Constantinescu [16] found $x / D \sim 1.60$ at the near-free surface; and Kirkil and Constantinescu [16] reported $x / D \sim 1.20$ at the near-bed. By comparing the length of the recirculation zone $(x / D \sim 1.20-1.6)$ for a single cylinder, it is clear that the downstream cylinder influences the longer recirculation zone midstream in S1. The smaller recirculation zone downstream in S1 and S2 are affected by the upstream cylinder. Similarly, Lin et al. [11] found that the upstream cylinder substantially altered the near-wake flow behind the downstream cylinder for $L / D=1.15$ to 5.10 at a cylinder Reynolds number $\left(R e_{D}\right)=1 \times 10^{4}$. As the flow approached the downstream (away from the recirculation zone), a rapid increase in velocity and the profiles approaching developed from $x / D=3.80$ in S3 midstream, $x / D=3.20$ in S1, and 3.80 in S2 and S3 downstream. In midstream, the flow deceleration was evident as it approached the downstream cylinder.
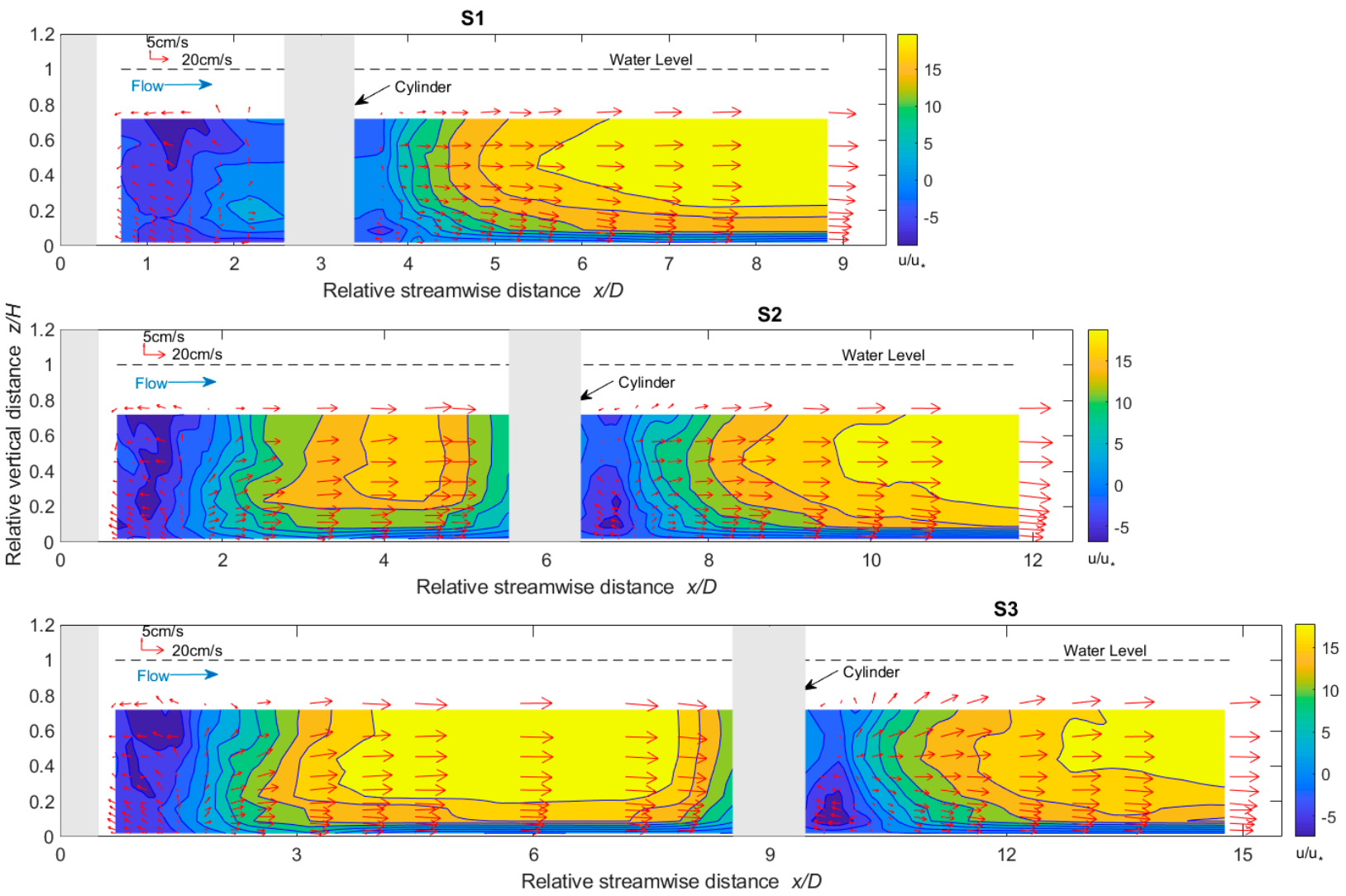

Figure 3. Velocity vector and isocontours of dimensionless streamwise velocity $(u / u *)$ on the symmetry plane for the 3 experimental scenarios S1, S2, and S3, combining both midstream and downstream. 
Figure 4 shows the variation of dimensionless depth averaged streamwise velocity $\left(u_{d} / u_{*}\right)$ along the plane of symmetry both midstream and downstream. Midstream, $u_{d} / u^{*}=0$ at $x / D=2.00$ in S1 and at $x / D=1.70$ in S2 and S3 (Figure 4a). In S1, the flow recirculation zone occupied almost the full space midstream, where $u_{d} / u_{*}$ varied from -5.20 to 0.40 . In $\mathrm{S} 2$, a rapid increase was found in $u_{d} / u^{*}=-4.40$ at $x / D=1.10$ to $u_{d} / u^{*}=13.20$ at $x / D=3.20$; then the velocity slowed, and the maximum $u_{d} / u^{*}=14.10$ occurred at $x / D=4.50$. Finally, the velocity dipped as the flow approached the downstream cylinder. In S3, similar to S2, $u_{d} / u_{*}$ increased sharply from -5.10 at $x / D=0.90$ to 14.30 at $x / D=3.20$. The rate of increase slowed and the maximum $u_{d} / u_{*}=16.60$ occurred at $x / D=7.20$; thereafter it decreased as the flow approached the downstream cylinder. In contrast to midstream, $u_{d} / u^{*}=0$ at $x / D 0.80,1.10$, and 1.20 in S1, S2, and S3, respectively (Figure $4 \mathrm{~b}$ ). The variation patterns of $u_{d} / u *$ in all three scenarios were similar, with a rapid increase in $u_{d} / u_{*}$ (varying from -3.80 to 17.40 ) between $x / D=0.70$ and $x / D=3.80$ and values then remaining almost constant. Interestingly, downstream the value of $u_{d} / u_{*}$ slightly decreased from S1 to S3 due to the effect of their corresponding recirculation lengths, which increased from S1 $(x / D \sim 0.90)$ to S3 $(x / D \sim 1.30)$ (as discussed above). Therefore, Figures 3 and 4 show that the downstream cylinder controls the flow recirculation length midstream in S1 and has zero interruption in S2 and S3.

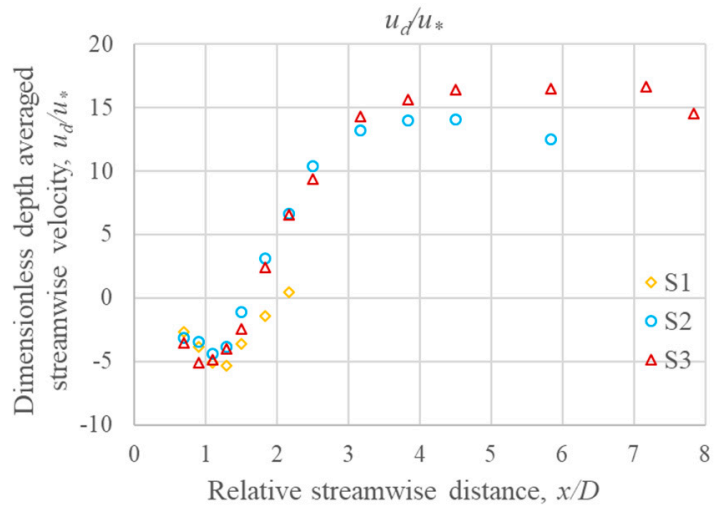

(a)

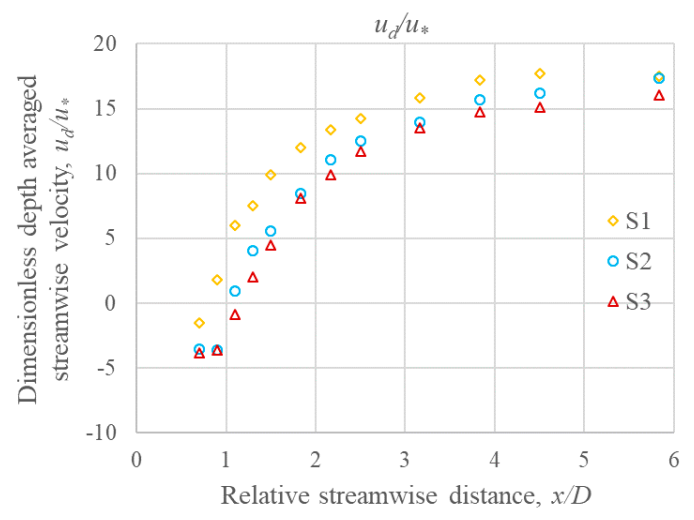

(b)

Figure 4. Variation of dimensionless depth-averaged streamwise velocity $\left(u_{d} / u_{*}\right)$ in the symmetry plane: (a) midstream and (b) downstream.

\subsection{Turbulent Flow Characteristics}

The time-averaged turbulent metrics of turbulent kinetic energy, $k_{\mathrm{e}}$, which characterizes the intensity of the turbulence, and the resultant Reynolds shear stress, $\tau_{p}$, which may promote sediment movement, are analyzed here to understand the turbulence flow characters better. Figure 5 shows the vertical profiles of dimensionless $k_{e} / u *^{2}$ in the symmetry plane for scenarios S1, S2, and S3 both midstream and downstream. Both midstream and downstream, the elevated $k_{e} / u_{*}^{2}$ grew with the streamwise distance until the end of the recirculation zone. With a further streamwise distance, despite the obvious reduction, the $k_{e} / u *^{2}$ remained at a relatively high level. The pronounced $k_{e} / u_{*}^{2}$ downstream of the cylinders is attributed to large-scale vortices generated in the recirculation region [25].

In order to acquire a better understanding, the variations of maximum $k_{e} / u^{2}$ for the plane of symmetry are also plotted in Figure $6 \mathrm{a}$ for midstream and in Figure $6 \mathrm{~b}$ for downstream. Midstream, it is apparent that the $k_{e}$ keeps on increasing as the flow progresses. The highest $k_{e} / u_{*}^{2}=154$ was observed at $x / D=1.83$ in S1, while $k_{e} / u_{*}{ }^{2}=152$ was observed at $x / D=1.83$ in S2, and $k_{e} / u^{2}=157$ was observed at $x / D=2.17$ in S3. In all scenarios, a rapid increase in $k_{e} / u^{2}$ (by about 3.9 times of the lowest $k_{e} / u^{2}$ ) was found over a short longitudinal distance $(x / D=0.70)$ to 2.17 . After that, the peak $k_{e} / u *^{2}$ gradually decreased by about 4.5 times over a distance $x / D=2.17$ to 7.83 in S3. Downstream, the highest $k_{e} / u_{*}^{2}=156$ was observed at $x / D=0.90$ in $S 1, k_{e} / u_{*}^{2}=159$ was observed at 
$x / D=1.30$ in S2, and $k_{e} / u^{*}{ }^{2}=108$ was observed at $x / D=1.10$ in S3. In all scenarios, a rapid increase in $k_{e} / u_{*}^{2}$ (by about 1.6, 2.1, and 2.8 times the lowest $k_{e} / u^{2}$ ) over a short longitudinal distance $x / D=0.70$ to $0.90,0.70$ to 1.30 , and 0.70 to 1.10 in S1, S2, and S3, respectively, was observed. After that, the $k_{e} / u_{*}^{2}$ peaks gradually decreased by about 4.3 , 4.4 , and 3.9 times over a distance $x / D=0.90$ to $5.83,1.30$ to 5.83 , and 1.10 to 5.83 in S1, S2, and S3, respectively. Therefore, peaks of $k_{e} / u_{*}{ }^{2}$ in both midstream and downstream for all three scenarios occurred near the end of their corresponding recirculation zones, except for S3 midstream where the peak occurred outside the recirculation zone.
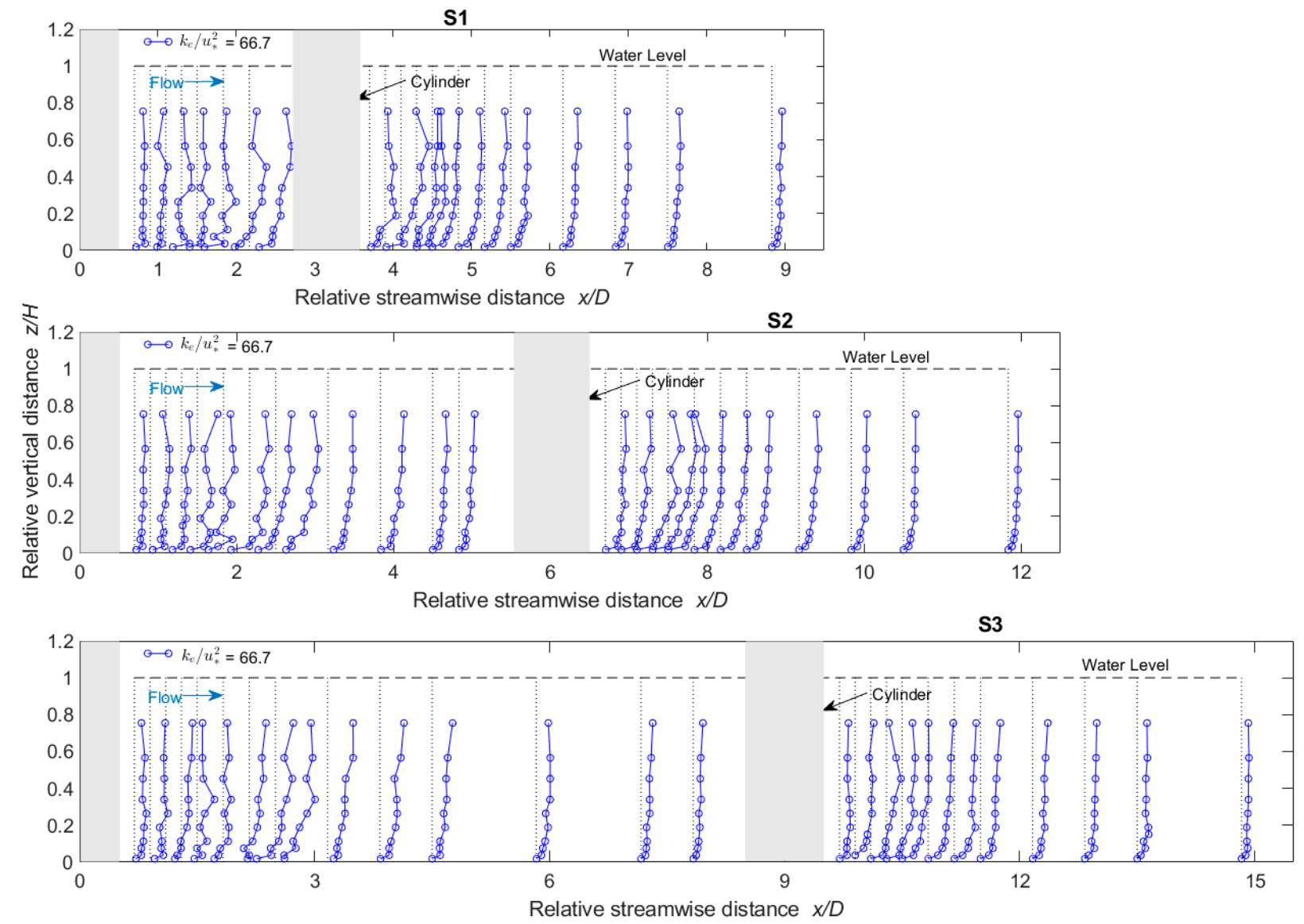

Figure 5. Vertical profiles of dimensionless turbulent kinetic energy $\left(k_{e} / u^{2}\right)$ in the symmetry plane for the 3 experimental scenarios S1, S2, and S3, combining both midstream and downstream.

It can also be observed from Figure 6 that the approaching $k_{\mathrm{e}}$ towards the downstream cylinder had a significant influence on the $k_{\mathrm{e}}$ just downstream of this cylinder. For S1, S2, and S3, the $k_{e} / u^{*}$ just upstream of downstream cylinder ended with 150,56 , and 35 , respectively, and their corresponding $k_{e} / u^{2}$ downstream started with 95,74 , and 38, indicating that the $k_{\mathrm{e}}$ downstream increased with the increased $k_{\mathrm{e}}$ midstream.

Figure 7 shows the vertical profiles of dimensionless resultant Reynolds shear stress $\left(\tau_{p} / u^{*}\right)$ in the plane of symmetry for S1, S2, and S3 both midstream and downstream. It is apparent that $\tau_{p} / u_{*}^{2}$ dominated near the recirculation zone for all scenarios. The magnitude of vertical profiles outside of the recirculation zone and in farther downstream locations was generally low and slightly elevated the near-free surface. Figure 8 shows the variations of maximum $\tau_{p} / u_{*}{ }^{2}$ for the plane of symmetry midstream and downstream, which are relatively chaotic as compared to the variation of $k_{e} / u^{2}$ with $x / D$.

Midstream, the highest $\tau_{p} / u^{*}{ }^{2}=15$ was observed at $x / D=1.83$ in S1 and $\tau_{p} / u_{*}{ }^{2}=14$ was observed at $x / D=2.50$ in S2 and S3 (Figure 8a). Similarly, downstream the highest $\tau_{p} / u^{2}{ }^{2}=13$ was observed at $x / D=1.10$ in $S 1$, where a value of 19 was observed at $x / D=1.30$ in $S 2$, and a value of 17 was obtained at $x / D=1.50$ in S3 (Figure 8b). Though the 
plots are scattered, in general the lowest values of $\tau_{p} / u^{2}{ }^{2}$ just downstream of the cylinders increase rapidly in the streamwise distance, and the values reach a peak in or outside the recirculation region; thereafter the values gradually decrease with the downstream distance. In all three scenarios, the peaks of $\tau_{p} / u_{*}{ }^{2}$ in downstream are higher than those midstream.

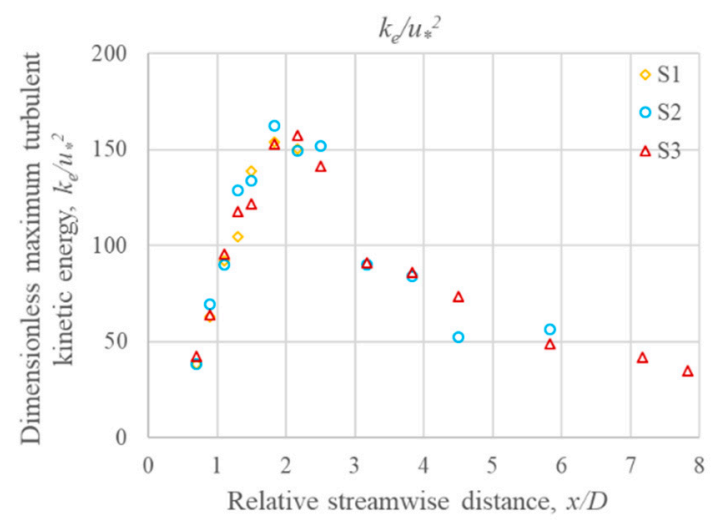

(a)

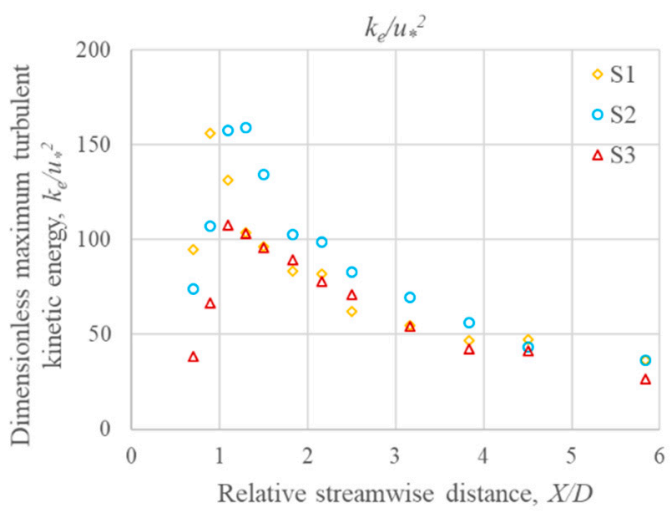

(b)

Figure 6. Variation of dimensionless maximum turbulent kinetic energy $\left(k_{e} / u_{*}^{2}\right)$ in the symmetry plane: (a) midstream and (b) downstream.
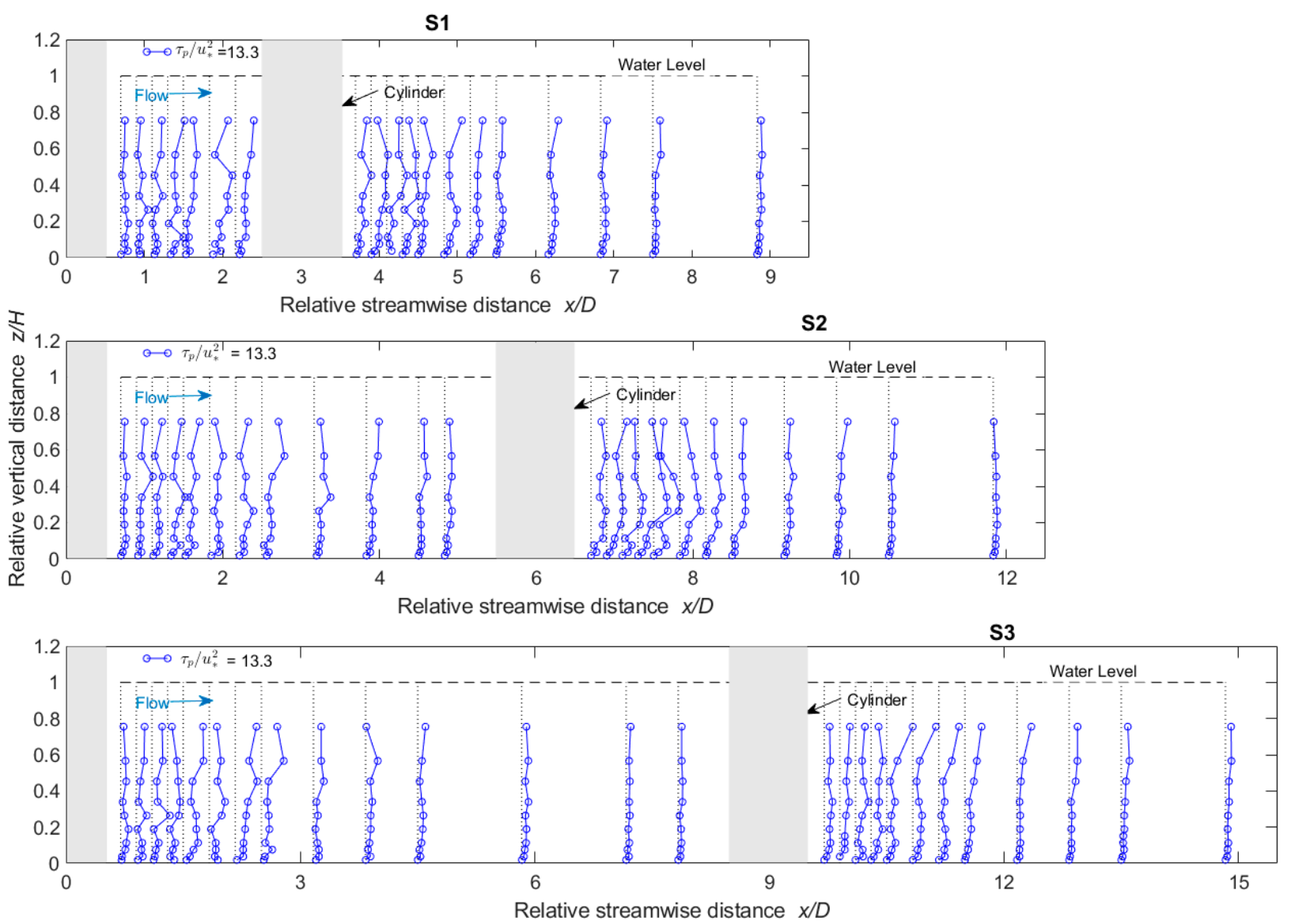

Figure 7. Vertical profiles of dimensionless resultant Reynolds shear stress $\left(\tau_{p} / u_{*}^{2}\right)$ in the symmetry plane for the 3 experimental scenarios S1, S2, and S3, combining both midstream and downstream. 


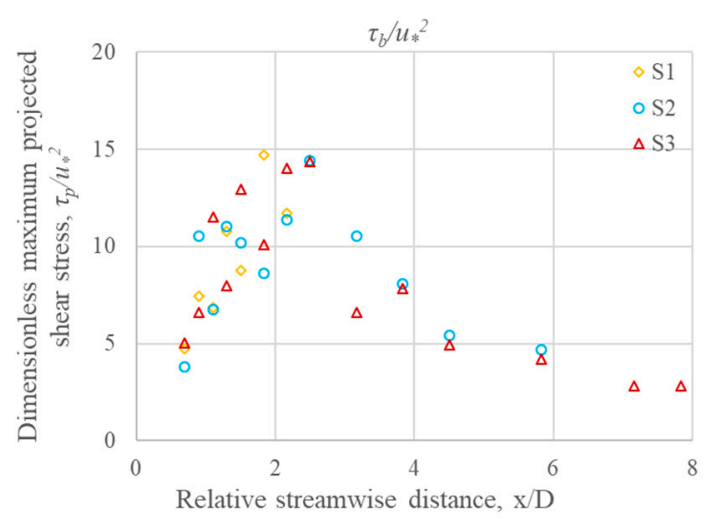

(a)

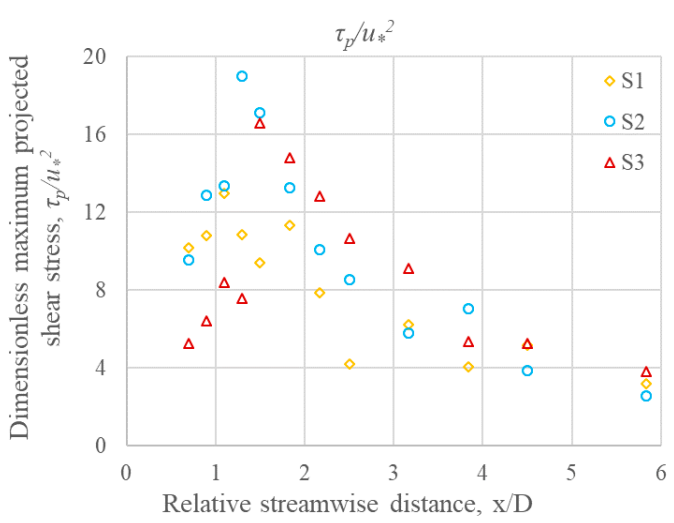

(b)

Figure 8. Variation of dimensionless maximum projected Reynolds shear stress $\left(\tau_{p} / u_{*}^{2}\right)$ in the symmetry plane: (a) midstream and (b) downstream.

\subsection{Near-Bed Shear Stress}

The spatial distribution of the dimensionless maximum near-bed shear stress $\left(\tau_{b}^{*}\right)$ is of great importance in the presence of cylinders that significantly change the local flow hydrodynamics, as plotted in Figure 9 using the Reynolds (Equation (3)), TKE (Equation (4)), and modified TKE (Equation (5)) methods.

Apparently, midstream the results for all three scenarios exhibit better trends along with the relative streamwise distance $(x / D)$ than downstream for all methods (Equations (3)-(5)). The peak values of $\tau_{b}^{*} \sim 10,20$, and 5.5 for the Reynolds, TKE, and the modified TKE methods, respectively, occurred at $x / D=1.30-1.83$ midstream, and downstream the peak values of $\tau_{b}^{*} \sim 9,19$, and 4.5 for the corresponding methods occurred at $x / D=1.10-1.30$. Therefore, the slightly elevated (5-20\%) peaks of $\tau_{b}^{*}$ occurred over an extended length of about $0.5 \mathrm{D}$ midstream, in contrast to the peaks of $\tau_{b}^{*}$ downstream, which occurred over a short length of 0.2D. Following the length of the flow recirculation zone (as discussed above), the peaks of $\tau_{b}^{*}$ mostly occurred at the end of the recirculation zone or the reattachment point. Studies have reported an increased Reynolds shear stress and TKE downstream of a submerged boulder near the bed due to the large-scale vortices in the wake zone $([26,27]$, and others). According to the trends both midstream and downstream for all methods, the lowest values of $\tau_{b}^{*}$ just downstream of the cylinders increased rapidly in the downstream distance and the values attained a peak in the recirculation region; thereafter they gradually decreased as the flow approached the downstream distance. As expected, it should be noted that trends in Figure 9 are very similar to the variations in maximum $k_{e} / u_{*}^{2}$ and $\tau_{p} / u_{*}^{2}$ with the relative streamwise distance $(x / D)$ in Figures 6 and 8.

Both midstream and downstream the variations of $\tau_{b}^{*}$ with $x / D$ using the three methods deviated from each other (Figure 9). The highest $\tau_{b}^{*}$ values were 10, 20 and 5.5 for the Reynolds, TKE, and modified TKE methods, respectively. The highest value of $\tau_{b}^{*}$ in the modified TKE method was exceptionally low (about 70\%) compared to the TKE method. The reason for this low value may be a less significant velocity fluctuation in the vertical direction $\left(w^{\prime}\right)$ in the calculation of the modified TKE shear stress. Similarly, the highest value of $\tau_{b}^{*}$ in the Reynolds method was about $50 \%$ less than that in the TKE method. 

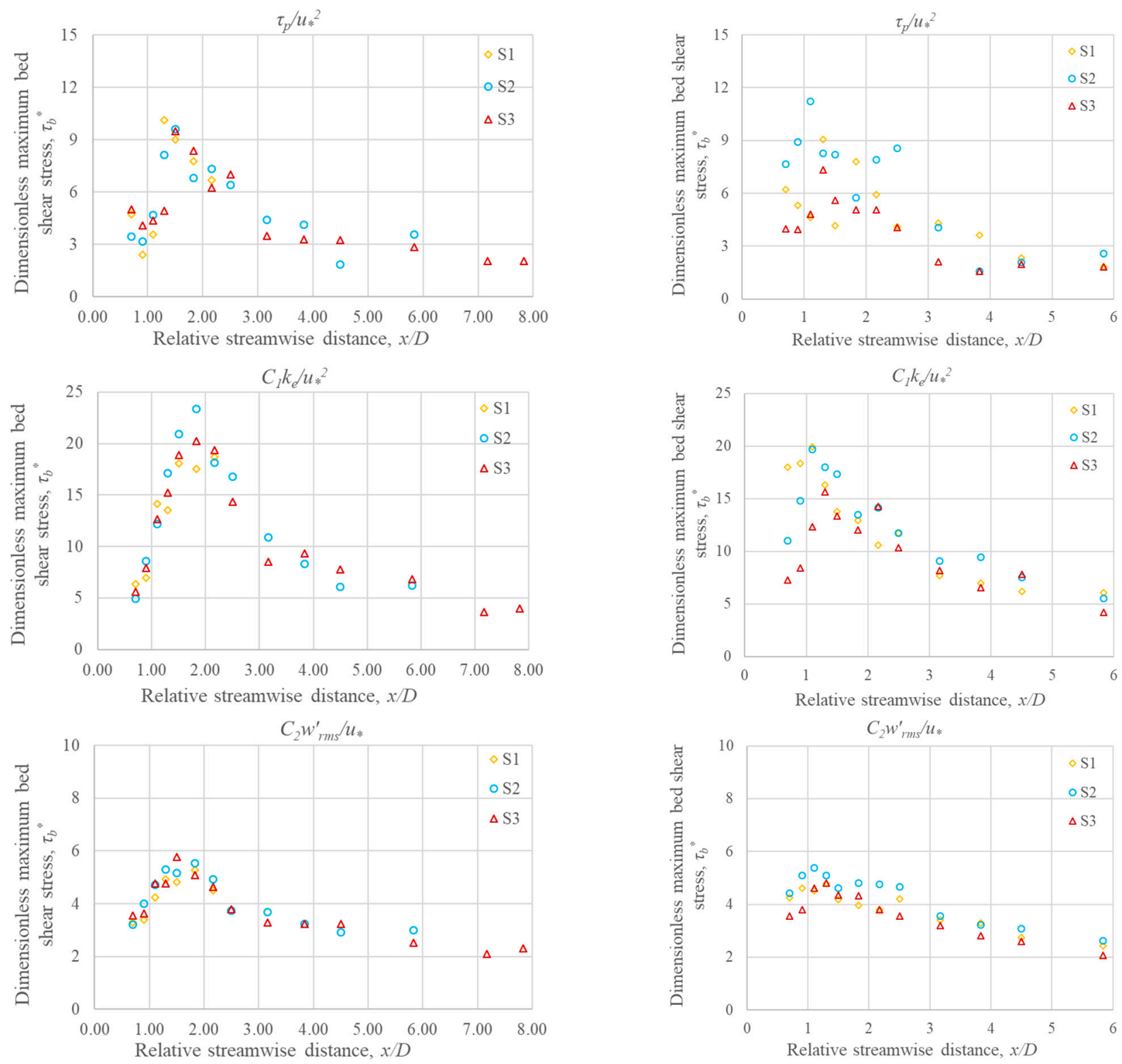

(a)

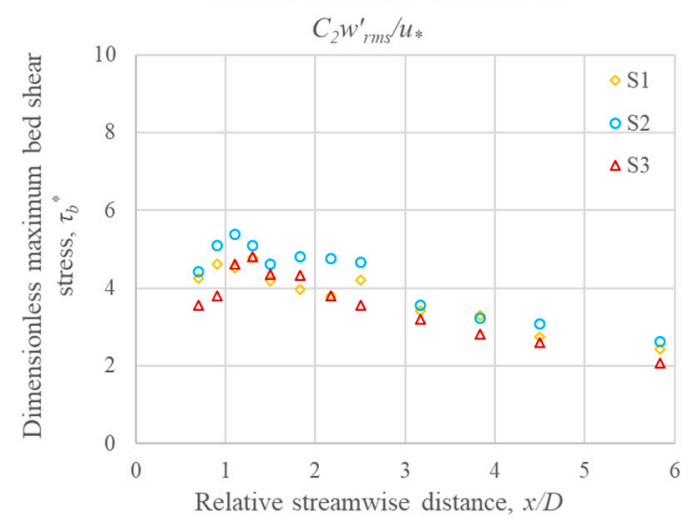

(b)

Figure 9. Variation of dimensionless maximum near-bed shear stress $\left(\tau_{b}^{*}\right)$ using the Reynolds (Equation (3)), TKE (Equation (4)), and modified TKE (Equation (5)) methods in the symmetry plane: (a) midstream and (b) downstream.

\section{Conclusions}

The experimental study examined the detailed flow fields around a pair of cylinders in a tandem arrangement placed at three different $\mathrm{c} / \mathrm{c}$ spacings along the plane of symmetry in an open channel. Mean and turbulent flow fields between two cylinders as well as in the wake of downstream cylinders were investigated using an ADV. The following concluding remarks are extracted from the results of this study.

- The analysis of vertical profiles of streamwise normalized velocity revealed that the rate of flow development downstream was faster than that midstream. The midstream profiles were influenced by the downstream cylinder in all scenarios.

- The flow recirculation zones midstream $(x / D \sim 1.50-1.80)$ were larger and stronger than those downstream $(x / D \sim 0.90-1.30)$. The longest recirculation zone in $\mathrm{S} 1$ midstream was affected by the downstream cylinder, and there was zero interruption from the downstream cylinder in S2 and S3. 
- The length of the recirculation zone increased with the increase in c/c spacing of cylinders. The rate of flow development to achieve cross-sectional mean velocity became slower with increasing $\mathrm{c} / \mathrm{c}$ spacing.

- The maximum turbulent kinetic energy for all three scenarios occurred approximately near the end of their respective recirculation zones. The midstream $k_{\mathrm{e}}$ had a significant influence on the $k_{\mathrm{e}}$ in downstream.

- The variation of resultant Reynolds shear stress was relatively chaotic as compared to the turbulent kinetic energy. The maximum shear stresses occurred within the recirculation zone for all scenarios.

- The peaks of dimensionless maximum near-bed shear stress $\left(\tau_{b}^{*}\right)$ were higher $(5-20 \%)$ and occurred over an extended length of about $0.5 \mathrm{D}$ in midstream as compared to the peaks of $\tau_{b}^{*}$ downstream, which occurred over a short length of $0.2 \mathrm{D}$. The highest value of $\tau_{b}^{*}$ in the TKE method was about $50 \%$ and $70 \%$ higher than that in the Reynolds and modified TKE methods, respectively.

This study's results may be helpful for developing computational fluid dynamic models for simulation flows around similar obstructions such as bridge piers. Moreover, the results can be used to understand and interpret sediment transport, scour, and fish habitat assessment in natural rivers with similar structures. Further studies are recommended to examine the effects of Reynolds number on flow characteristics and turbulent events effects on sediment mobility for a tandem arrangement of the cylinders.

Author Contributions: Conceptualization, H.Z. and A.B.M.B.; methodology, H.Z., A.B.M.B., and S.N.N.; formal analysis, H.Z., A.B.M.B., and S.N.N.; investigation, H.Z. and S.N.N.; resources, H.Z.; writing-original draft preparation, H.Z. and A.B.M.B.; writing-review and editing, H.Z. and A.B.M.B.; supervision, H.Z.; project administration, H.Z.; All authors have read and agreed to the published version of the manuscript.

Funding: The third author was provided with financial support from the Bangladesh University of Engineering and Technology (BUET).

Institutional Review Board Statement: Not applicable.

Informed Consent Statement: Not applicable.

Data Availability Statement: Some or all data, models, or code generated or used during the study are available from the corresponding author by request.

Acknowledgments: The authors wish to thank laboratory attendants for their invaluable help in the lab measurements. The manuscript benefitted from the constructive comments of anonymous reviewers.

Conflicts of Interest: The authors declare no conflict of interest.

\section{References}

1. Zhou, Y.; Yiu, M.W. Flow structure, momentum and heat transport in a two-tandem-cylinder wake. J. Fluid Mech. 2006, 548, 17-48. [CrossRef]

2. Heidari, M. Wake Characteristics of Single and Tandem Emergent Cylinders in Shallow Open Channel Flow. Ph.D. Thesis, University of Windsor, Windsor, ON, Canada, 2016. Available online: https://scholar.uwindsor.ca/etd/5733 (accessed on 24 January 2021).

3. Nosier, M.A.; Elbaz, A.R.; Fetouh, T.A.; El-Gabry, L.A. Characteristics of turbulent wakes generated by twin parallel cylinders. J. Fluids Eng. 2012, 134, 121201. [CrossRef]

4. AlQadi, I.; AlHazmy, M.; Al-Bahi, A.; Rodi, W. Large eddy simulation of flow past tandem cylinders in a channel. Flow Turbul. Combust. 2015, 95, 621-643. [CrossRef]

5. Shamloo, H.; Rajaratnam, N.; Katopodis, C. Hydraulics of simple habitat structures. J. Hydraul. Res. 2001, 39, 351-366. [CrossRef]

6. Sadeque, M.F.; Rajaratnam, N.; Loewen, M.R. Shallow turbulent wakes behind bed-mounted cylinders in open channels. J. Hydraul. Res. 2009, 47, 727-743. [CrossRef]

7. Rostamy, N.; Sumner, D.; Bergstrom, D.J.; Bugg, J.D. Local flow field of a surface-mounted finite circular cylinder. J. Fluids Struct. 2012, 34, 105-122. [CrossRef]

8. Zdravkovich, M.M. The effects of the interference between circular cylinders in cross flow. J. Fluids Struct. 1987, 1, 239-261. [CrossRef] 
9. Sharman, B.; Lien, F.S.; Davidson, L.; Norberg, C. Numerical predictions of low Reynolds number flows over two tandem circular cylinders. Int. J. Numer. Meth. Fluids 2005, 47, 423-447. [CrossRef]

10. Papaioannou, G.V.; Yue, D.K.P.; Triantafyllou, M.S.; Karniadakis, G.E. Three-dimensionality effects in flow around two tandem cylinders. J. Fluid Mech. 2006, 558, 387-413. [CrossRef]

11. Lin, J.C.; Yang, Y.; Rockwell, D. Flow past two cylinders in tandem: Instantaneous and averaged flow structure. J. Fluids Struct. 2002, 16, 1059-1071. [CrossRef]

12. Pinarbasi, A.; Pinar, E.; Akilli, H.; Ince, E. Shallow water experiments of flow past two identical square cylinders in tandem. Eur. J. Mech. B/Fluids 2015, 49, 100-107. [CrossRef]

13. Akilli, H.; Rockwell, D. Vortex formation from a cylinder in shallow water. Phys. Fluids 2002, 14, 2957-2967. [CrossRef]

14. Gao, Y.; Yu, D.; Tan, S.; Wang, X.; Hao, Z. Experimental study on the near wake behind two side-by-side cylinders of unequal diameters. Fluid Dyn. Res. 2010, 42, 055509. [CrossRef]

15. Kirkil, G.; Constantinescu, G. A numerical study of the laminar necklace vortex system and its effect on the wake for a circular cylinder. Phys. Fluids 2012, 24, 073602. [CrossRef]

16. Kirkil, G.; Constantinescu, G. Effects of cylinder Reynolds number on the turbulent horseshoe vortex system and near wake of a surface-mounted circular cylinder. Phys. Fluids 2015, 27, 075102. [CrossRef]

17. Nezu, I.; Nakagawa, H. Turbulence in Open-Channel Flows; Balkema: Rotterdam, The Netherlands, 1993.

18. Baki, A.B.M.; Zhang, W.; Zhu, D.Z.; Rajaratnam, N. Flow structures in the vicinity of a submerged boulder within a boulder array. J. Hydraul. Eng. 2016, 143, 04016104. [CrossRef]

19. Biron, P.M.; Robson, C.; Lapointe, M.F.; Gaskin, S.J. Comparing different methods of bed shear stress estimates in simple and complex flow fields. Earth Surf. Process. Landf. J. Br. Geomorphol. Res. Group 2004, 29, 1403-1415. [CrossRef]

20. Strom, K.B.; Papanicolaou, A.N. ADV measurements around a cluster microform in a shallow mountain stream. J. Hydraul. Eng. 2007, 12, 1379-1389. [CrossRef]

21. Wahl, T.L. WinADV Version 2.028: A Windows-Based Viewing and Post-Processing Utility for ADV Files; User's Manuals and On-Line Help Provided with the Software. 2002. Available online: http://www.usbr.gov/wrrl/twahl/winadv/ (accessed on 24 January 2021).

22. Goring, D.G.; Nikora, V.I. Despiking acoustic Doppler velocimeter data. J. Hydraul. Eng. 2002, 128, 117-126. [CrossRef]

23. Kim, S.C.; Friedrichs, C.T.; Maa, J.Y.; Wright, L.D. Estimating bottom stress in tidal boundary layer from acoustic Doppler velocimeter data. J. Hydraul. Eng. 2000, 126, 399-406. [CrossRef]

24. Papanicolaou, A.N.; Kramer, C.M.; Tsakiris, A.G.; Stoesser, T.; Bomminayuni, S.; Chen, Z. Effects of a fully submerged boulder within a boulder array on the mean and turbulent flow fields: Implications to bedload transport. Acta Geophys. 2012, 60, 1502-1546. [CrossRef]

25. Zexing, X.; Chenling, Z.; Qiang, Y.; Xiekang, W.; Xufeng, Y. Hydrodynamics and bed morphological characteristics around a boulder in a gravel stream. Water Supply 2020, 20, 397-407. [CrossRef]

26. Dey, S.; Sarkar, S.; Bose, S.K.; Tait, S.; Castro-Orgaz, O. Wall-wake flows downstream of a sphere placed on a plane rough wall. J. Hydraul. Eng. 2011, 137, 1173-1189. [CrossRef]

27. Baki, A.B.M.; Zhu, D.Z.; Rajaratnam, N. Turbulence characteristics in a rock-ramp-type fish pass. J. Hydraul. Eng. 2015, 141, 04014075. [CrossRef] 